\title{
Effectiveness of Mental Health Intervention for Suicide Prevention
}

\author{
Nimesh G. Desai, MD. ${ }^{1}$, Vishi Sachdeva, MD. ${ }^{2 *}$, Aishwarya John, MD. ${ }^{1}$, \\ Kumar Gourav, MD. ${ }^{1}$, Gibson 0. Anugwom, MD., MSc. ${ }^{3}$
}

\author{
${ }^{1}$ Department of Psychiatry, Institute of Human Behavior and Allied Science, Delhi, India. \\ ${ }^{2}$ Adesh Institute of Medical Sciences and Research, Bathinda, India. \\ ${ }^{3}$ Houston Behavioral Healthcare Hospital, Houston, Texas, USA. \\ *Corresponding author details: Vishi Sachdeva, MD; Vishisachdeva@gmail.com
}

\begin{abstract}
Background: Various mental health interventions like priority in-patient care, ECT, psychotherapies, pharmacotherapies, etc. have been tried throughout the world to decrease the morbidity and mortality associated with suicide behavior.

Aims: To establish the effectiveness of mental health intervention for preventing suicide, for those at risk and to understand the perception of patients and family members about the usefulness of interventions for preventing suicide.

Material and methods: The patients admitted to the Psychiatry Intensive Care Unit (PICU) at the Institute of Human Behavior and Allied Sciences (IHBAS), in view of suicide behavior, during the 12 months period from 1st July 2018 to 30 th June 2019 were included in the study. A target population of 88 patients was taken up for cross-sectional follow-up assessment. They were assessed for suicide behavior for the period of 12 months prior to and subsequent to the hospitalization.

Results: A statistically significant difference was found in both, the number of patients attempting suicide before and after hospitalization $(\mathrm{N}=88$, Chi-square $=.2$, p-value $<0.04)$, as well as in the mean number of attempts before and after hospitalization $(\mathrm{N}=88$, $\mathrm{p}$-value $<0.01)$. Also, three fourth of patients/family members were completely satisfied with the care provided while the remaining one-fourth were only partially satisfied. Conclusion: This study has established not only the usefulness of timely mental health intervention for the prevention of suicide as perceived by family or patients but also provides statistical evidence for the effectiveness of such mental health interventions.
\end{abstract}

Keywords: Suicide; mental health intervention; hospitalization; India; psychiatry

\section{INTRODUCTION}

Suicide is a major public health problem worldwide with complex multi-factorial origins. More than 800,000 lives worldwide are lost to suicide every year and Asia accounts for more than $60 \%$ of such deaths. [1, 2] The estimated suicide-related death rate in India is $21 / 100,000$, which is near twice the global average $(11.4 / 100,000)$ and translates into more than 230,000 lives lost annually. [3]

In a 13-year follow-up study of a community sample in Baltimore, Maryland, it was found that people who contemplate suicide were over six times more likely to report suicide attempt over the course of a 13-year followup interval. This finding suggests that experiencing suicidal ideation represents an elevated risk of attempting suicide at some point in the future. [4]

Psychiatric illness accounts for a large majority of suicides and suicide attempts; numbers are at least 10 times as high as in the general population. The reported percentage of completed suicides in this context ranges between $60 \%$ and $98 \%$ of all suicides. [5] Amongst psychiatric patients, the risk of suicide behavior (suicidal ideation and suicide attempt) is seen more in patients who had previously reported thoughts of self-harm or suicide, patients recently discharged from treatment centers, and patients who were previously hospitalized for risk of suicide behavior. ${ }^{6,7,8]}$ Various mental health interventions such as increased priority in patient care, modified electroconvulsive therapy (ECT), psychotherapies, antidepressants, etc. have been tried throughout the world, with various efficacy to decrease the morbidity and mortality associated with suicide behavior. [14]

The efficacy of modified ECT in decreasing current suicide behavior in individual patients and its usefulness as a clinical strategy has been well recorded in literature but there are very few studies that assess its long-term effect in the reduction of suicide behavior. A study by Avery et al. in the year 1969 assessed suicidal behavior in the 6 months after the treatment of depression in 519 patients and found that suicide attempts were recorded in $0.8 \%$ of the ECT-treated patients as compared to $11.2 \%$ of those who had received antidepressant treatment alone.[9]

Findings of the meta-analysis done by Crawford et al. which included 18 studies reported no evidence that psychosocial interventions had a notable effect on the likelihood of subsequent suicide attempts. [10] 
However, strong evidence for the effectiveness of counseling and psychotherapy emerged from the meta-analysis done by Winter et al., in the year 2013, where 44 of the 54 studies analyzed showed the benefits of psychological interventions in reducing the risks of suicide behavior. [11]

Although it is generally believed and practiced that hospitalization is a critical strategy for the management of patients at risk of suicide, very few studies have been conducted to examine its effectiveness. A study by Kapur et al. done in Manchester, U.K. in 2015 showed that psychiatric inpatient admission was associated with reduced mortality risks in men, older adults, and those with a history of self-harm as compared to only psychosocial assessment, admission in a medical facility or follow up in a community mental health facility.[12]

A two-year prospective study, done by Hayashi et al., assessed the post-hospitalization course and predictive signs of suicidal behavior. They assessed 106 patients who were admitted to a psychiatric hospital with a history of self-harm and suicide attempts, in Tokyo in the year 2012. They described their findings at the end of 1 year and 2 years separately. At the end of one year, they found that $2.8 \%$ of patients had completed suicide and $27 \%$ reported suicide attempts while at the end of 2 years it was found to be $6 \%$ and $38 \%$ respectively. ${ }^{[13]}$

A one-year retrospective cohort study, using 4 Mediclaim databases, was conducted by Cepeda et al., in the year 2015 in the United States which included 121,065 patients with depressive disorder who were hospitalized in view of suicidal behavior. In this study, hospitalization included both emergency room visits and in-patient treatment. The rate of rehospitalization for suicide behavior ranged from 7.96 to $11.24 \%$ at the end of the study. ${ }^{[8]}$ Both these studies did not assess the effectiveness of hospitalization as a strategy for the prevention of suicide behavior.

A meta-analysis by Hofstra et al. provided a comparative estimate of the effect of different types of suicide prevention interventions, based on 15 controlled studies, with 29,071 participants in various settings. The findings showed that suicide prevention interventions are effective in reducing the number of completed and attempted suicides. For attempted suicides, outpatient mental health interventions showed the highest effect size followed by admission to a psychiatric ward in a general hospital. Other interventions like emergency department visits showed lesser effect size. However, a combination of one or more methods and multilevel intervention showed the highest effect size. [14]

The usefulness of suicide prevention strategies including mental health intervention in the management of suicide behavior has been established but its effectiveness needs more exploration.

\section{OBJECTIVES}

1) To establish the effectiveness of mental health intervention for preventing suicide, for those at risk.

2) To understand the perception of patients and family members about the usefulness of intervention for preventing suicide.

\section{METHODOLOGY}

The patients admitted to the Psychiatry Intensive Care Unit (PICU) at the Institute of Human Behavior and Allied Sciences (IHBAS), in view of suicide behavior, during 12 months from 1 July 2018 to 30 June 2019 were included in the study. The admission record register was used to screen the case files of these patients. Among the 132 cases screened, 19 had a history of non-fatal deliberate self-harm,
8 were repeat admissions and 9 were incorrect entries. Hence, these 36 cases were excluded. The other 96 cases were included as the target study sample. All these patients were taken up for cross-sectional follow-up assessment in June-July 2020. These 96 patients/family members were contacted through telephone and Informed consent, including confidentiality for the follow-up assessment, was obtained. Appropriate intervention was provided based on the patient's clinical need at the time of follow-up assessment and acceptance of the offer by the patient/family. Eight patients/family members refused to give consent for the study, thus yielding a study sample of 88 patients. They were assessed for a period of 12 months subsequent to their discharge. This study was conducted in accordance with the Helsinki Declaration as revised in 1989.

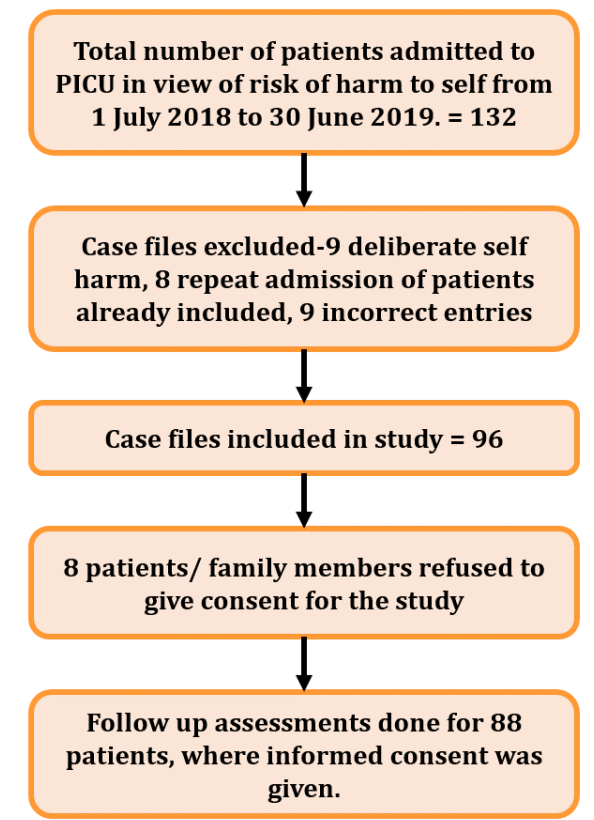

FIGURE 1: Flowchart depicting sample selection

Information was obtained using semi-structured pro forma over the telephone or on outpatient department (OPD) follow-ups and further supplemented by case file records. Although the patients were approached after 12-24 months of discharge, the assessment was done for a period of only the first 12 months after discharge. The exercise was taken up primarily to understand the effectiveness of the routine mental health interventions provided during admission.

\section{RESULTS}

The study sample of 88 patients consisted of 52 (59.1\%) males and $36(40.9 \%)$ females. The mean age was $35.23( \pm$ $14.3)$ years. $32(36.4 \%)$ of the patients were unmarried, 55 $(62.5 \%)$ were married and $1(1.1 \%)$ was divorced. The sample included 10 (11.4\%) students, 58 (65.9\%) unemployed individuals, 13 (14.8\%) unskilled workers, 6 (6.8\%) skilled and $1(1.1 \%)$ professional.

The diagnosis of Depression, as per ICD-10, was found in 62 (70.5\%) patients while other diagnoses like schizophrenia, postpartum psychosis, adjustment disorder, dissociative disorder, polysubstance abuse were found in 26 (29.5\%) patients. Fifty-three $(60.3 \%)$ patients were admitted in view of suicide attempt and the other 35 (39.7\%) patients in view of suicidal ideations.

On follow-up assessment, it was found that out of the 88 patients, $21(23.8 \%)$ patients had attempted suicide, 2 (2.3\%) patients completed suicide and $1(1.1 \%)$ patient died due to medical cause. For further analysis, the 2 patients who died by suicide were included with patients who had attempted suicide, and 1 patient who died of medical cause, 
had not attempted suicide in the post-discharge follow-up period, was thus included with patients who had not attempted suicide.
Thus, $23(26.1 \%)$ out of 88 patients were found to have attempted suicide during the one-year follow-up period.

TABLE 1: Comparison of number of patients attempting suicide one year before and after the index admission. $(n=88)$

\begin{tabular}{|c|c|c|c|c|c|c|}
\hline & & \multicolumn{3}{|c|}{$\begin{array}{l}\text { Number of patients who attempted suicide } \\
\text { in one year follow up after hospitalization }\end{array}$} & \multirow{2}{*}{$\begin{array}{c}\text { Chi-square } \\
\text { Test }\end{array}$} & \multirow{2}{*}{ p-value } \\
\hline & & Yes & No & Total & & \\
\hline \multirow{3}{*}{$\begin{array}{l}\text { Number of patients who } \\
\text { attempted suicide in the one } \\
\text { year prior to hospitalization }\end{array}$} & Yes & 18 & 35 & 53 & \multirow{3}{*}{4.2} & \multirow{3}{*}{$0.04^{*}$} \\
\hline & No & 5 & 30 & 35 & & \\
\hline & Total & 23 & 65 & 88 & & \\
\hline
\end{tabular}

Chi-square test was used

$*$ p value significant at $</=0.05$

Table 1 depicts the data of number of patients who attempted suicide in the one-year follow-up period after hospitalization, in the subgroups of patients who had/ had not attempted suicide, in the one year preceding the hospitalization. Out of 53 patients who had attempted suicide in the previous year, 18 patients attempted suicide in the follow-up period while out of 35 patients who had not attempted suicide in the previous year, 5 patients attempted suicide during the follow-up period. A statistically significant difference was found $(\mathrm{p}$-value $=$ 0.04 ), indicating that mental health intervention had a positive impact in reducing the number of suicide attempts post-treatment, in patients with suicide attempts prior to hospitalization.

TABLE 2: Comparison of mean number of suicide attempts in one year before and after admission $(\mathrm{n}=88)$

\begin{tabular}{|c|c|c|c|}
\cline { 2 - 3 } \multicolumn{1}{c|}{} & Before admission & After admission & P-value \\
\hline Mean no of attempts & $1.02 \pm 1.10$ & $0.28 \pm 0.50$ & \multirow{2}{*}{$<0.01 *$} \\
\cline { 1 - 3 } Median attempts & $1(0-2)$ & $0(0-1)$ & \\
\hline
\end{tabular}

Wilcoxon Signed Rank test was used

*p value significant at $</=0.05$

To examine the data in another manner, the mean number of suicide attempts in the total sample of 88 patients was compared for one year before the hospitalization $(1.02 \pm 1.10)$ and for the follow-up period of one year after $(0.28 \pm 0.50)$ the index admission.
This mirror image comparison was analyzed using the Wilcoxon Signed-Rank test and a statistically significant difference was found in favor of the post hospitalization period, again indicating that mental health intervention helped in decreasing suicide behavior.

TABLE 3: Association of clinical and treatment variables with number of suicide attempts during one year after the discharge $(\mathrm{N}=88)$

\begin{tabular}{|c|c|c|c|c|}
\hline Variable & Number(n) & Finding & No. of suicide attempts in the follow-up period & p-value \\
\hline \multirow{2}{*}{$\begin{array}{l}\text { PICU stay } \\
\text { (in weeks) }\end{array}$} & 62 & $<1$ week & $0.26 \pm 0.51$ & \multirow{2}{*}{0.29} \\
\hline & 26 & $>1$ week & $0.35 \pm 0.48$ & \\
\hline \multirow{2}{*}{ Modified ECT } & 40 & Yes & $0.23 \pm 0.42$ & \multirow{2}{*}{0.46} \\
\hline & 48 & No & $0.33 \pm 0.56$ & \\
\hline \multirow{2}{*}{$\begin{array}{c}\text { Non- } \\
\text { pharmacological } \\
\text { measures }\end{array}$} & 42 & Yes & $0.25 \pm 0.44$ & \multirow{2}{*}{0.86} \\
\hline & 46 & No & $0.28 \pm 0.50$ & \\
\hline \multirow[b]{2}{*}{ Diagnosis } & 62 & Depression & $0.26 \pm 0.51$ & \multirow{2}{*}{0.29} \\
\hline & 26 & Others & $0.35 \pm 0.48$ & \\
\hline \multirow[b]{2}{*}{ Follow-up } & 43 & Yes & $0.24 \pm 0.46$ & \multirow[b]{2}{*}{$0.04^{*}$} \\
\hline & 45 & No & $0.60 \pm 0.69$ & \\
\hline
\end{tabular}

${ }^{*}$ p-value significant at $</=0.0$ 
In table 3, Mann Whitney U test and Kruska Wallis $\mathrm{H}$ test were used to analyze the association of various clinical and treatment variables with suicide behavior, during the postdischarge one-year follow-up period. A statistically significant difference was found between patients who had followed up to the hospital after discharge and patients who had not. Other than this, no single intervention including modified ECT, non-pharmacological measures, or length of PICU stay was found to have a statistically significant difference in the patients who attempted suicide during the follow-up period and those who did not.

In our study, an attempt was made to understand the perception of patients/ family members regarding the usefulness of the mental health interventions provided during the admission and the post-discharge OPD followups. $75 \%$ of them were completely satisfied with the care and interventions provided and the remaining $25 \%$ were only partially satisfied.

\section{DISCUSSION}

In our study, out of 88 patients who had been admitted for suicide risk, 53 patients $(60.3 \%)$ had a history of suicide attempts preceding the index admission. At end of a oneyear follow-up, the number of suicide attempts showed a statistically significant reduction with 21 patients (23.8\%) attempting suicide and $2(2.3 \%)$ patients completing suicide, as shown in table 1 . Similar findings were reported in a study done by Hayasi et al., where 106 patients with suicidal behavior were assessed and at the end of 1 year, $27 \%$ of patients had attempted suicide while $2.8 \%$ of patients had completed suicide.

Cepeda et al. studied the recurrence of both suicidal ideation and attempts warranting readmission and found it to be $7.96 \%$ to $11.24 \%$. In our study, $8(9.09 \%)$ patients were readmitted, however, 23 patients $(26.1 \%)$ had attempted suicide in the one-year follow-up period. The lesser number of readmissions as compared to the number of reattempts during the follow-up period was probably due to the fact that some of them were not able to reach the hospital due to different reasons, including issues of logistics and accessibility.

Avery et al. compared 519 depressive patients receiving modified ECT, irrespective of whether they received antidepressants or not, with the patients receiving antidepressants only. On assessing the number of suicide attempts in the six months following discharge, they found a statistically significant reduction in the patients who had received modified ECT as compared to those who had not.

In our study, all patients received appropriate psychotropic medications (antipsychotics and/or antidepressants) and the choice of modified ECT was a clinical decision by the treating team. This study found no such difference in the frequency of suicide attempts in patients who received psychotropics alone or psychotropics with modified ECT.

Similarly, we found no difference in the frequency of suicide attempts in patients who had received different forms of psychotherapeutic interventions in addition to the physical forms of treatment (medication and/or ECT) as compared to those who had not. This contrasts with the finding of the meta-review by Winter et al., who found strong evidence for psychological intervention alone in reducing future suicide behavior, but in line with the finding of the meta-analysis by Crawford who found no significant role of psychological intervention. Unlike the studies analyzed by them, in our study patients received psychological intervention in addition to physical modes of treatment.
The study found a statistically significant association between OPD follow-up visits and reduction of suicide behavior. It may be inferred that regular OPD follow-up visits contributed to less likelihood of suicide attempts, but it is equally arguable that patients who were doing well in the follow-up period with a lesser number of attempts attended OPD more frequently. The factors affecting the association between suicide attempts and follow-up visits may be confounded by factors like achieving remission, therapeutic relationship, satisfaction with treatment, good social support, etc. The statistically significant association found by itself cannot be considered adequate to answer this issue.

In this study, the patients received a combination of various mental health interventions including intensive in-patient care, behavioral contracting, supportive therapy, disorderspecific pharmacotherapy, and modified ECT depending on the clinical need. This study did not find a significant association between specific interventions given during hospitalization and the suicide attempts in the follow-up period. However, the significant decrease in the number of patients who attempted suicide and the mean number of attempts in one year before and after hospitalization provides strong evidence for the effectiveness of multimodal mental health interventions. This finding was similar to the finding of the meta-analysis done by Hofstra et al., who found multi-modal interventions including outpatient services to be effective in reducing the number of future suicide attempts and suicides.

Further, this study also provides additional supportive evidence in the form of reported usefulness of mental health interventions for preventing suicide attempts as per the perceptions of the patient and family members and their satisfaction with the in-patient care and OPD followups. Three-fourths (75\%) of patients and family members were fully satisfied while one-fourth (25\%) were partially satisfied.

Amongst the various suicide prevention strategies emphasized in clinical and social context, the relevance and importance of effective mental health interventions are often taken for granted, or even overlooked. This study has provided qualitative and quantitative proof for suicide prevention being achieved through timely mental health treatment services, alongside other preventive strategies.

\section{CONCLUSION}

This study has established not only the usefulness of timely mental health interventions for the prevention of suicide as perceived by family members or patients but also provides statistical evidence for the effectiveness of such mental health interventions. Further studies are necessary to evaluate the effectiveness of other treatment methods such as Transcranial Magnetic Stimulation (TMS) and Ketamine therapy use in managing psychiatric conditions associated with suicide e.g., depression.

\section{REFERENCES}

[1] World Health Organization. (2014, August). Preventing Suicide: A global imperative.

[2] Beautrais A. L. (2006). Suicide in Asia. Crisis, 27(2), 55-57. https://doi.org/10.1027/0227-5910.27.2.55

[3] Bertolote J.M., \& Fleischmann A. (2002). A global perspective in the epidemiology of suicide. Suicidologi, 7, 6-8. http://doi.org/10.5617/suicidologi.2330 
[4] Kuo, W. H., Gallo, J. J., \& Tien, A. Y. (2001). Incidence of suicide ideation and attempts in adults: the 13year follow-up of a community sample in Baltimore, Maryland. Psychological medicine, 31(7), 1181-1191. https://doi.org/10.1017/s0033291701004482

[5] Ferrari, A. J., Norman, R. E., Freedman, G., Baxter, A. J., Pirkis, J. E., Harris, M. G., Page, A., Carnahan, E., Degenhardt, L., Vos, T., \& Whiteford, H. A. (2014). The burden attributable to mental and substance use disorders as risk factors for suicide: findings from the Global Burden of Disease Study 2010. PloS one, 9(4), e91936.https://doi.org/10.1371/journal.pone.0091 936

[6] Simon, G. E., Coleman, K. J., Rossom, R. C., Beck, A., Oliver, M., Johnson, E., Whiteside, U., Operskalski, B., Penfold, R. B., Shortreed, S. M., \& Rutter, C. (2016). Risk of suicide attempt and suicide death following completion of the Patient Health Questionnaire depression module in community practice. The Journal of clinical psychiatry, 77(2), 221-227. https://doi.org/10.4088/JCP.15m09776

[7] Forte, A., Buscajoni, A., Fiorillo, A., Pompili, M., \& Baldessarini, R. J. (2019). Suicidal Risk Following Hospital Discharge: A Review. Harvard review of psychiatry, 27(4),209-216. https://doi.org/10.1097/HRP.0000000000000222

[8] Cepeda, M. S., Schuemie, M., Kern, D. M., Reps, J., \& Canuso, C. (2020). Frequency of rehospitalization after hospitalization for suicidal ideation or suicidal behavior in patients with depression. Psychiatry research, 285, 112810. Advance online publication. https://doi.org/10.1016/j.psychres.2020.112810

[9] Avery, D., \& Winokur, G. (1976). Mortality in depressed patients treated with electroconvulsive therapy and antidepressants. Archives of general psychiatry, 33(9),1029-1037. https://doi.org/10.1001/archpsyc.1976.017700900 19001
[10] Crawford, M. J., Thomas, O., Khan, N., \& Kulinskaya, E. (2007). Psychosocial interventions following selfharm: systematic review of their efficacy in preventing suicide. The British journal of psychiatry: the journal of mental science,190, 11-17. https://doi.org/10.1192/bjp.bp.106.025437

[11] Winter, D., Bradshaw, S., Bunn, F.,\& Wellsted, D. (2013) A systematic review of the literature on counselling and psychotherapy for the prevention of suicide: 1 . Quantitative outcome and process studies. Counselling and Psychotherapy Research,13, 164-183. https://doi.org/10.1080/14733145.2012.761717.

[12] Kapur, N., Steeg, S., Turnbull, P., Webb, R., Bergen, H., Hawton, K., Geulayov, G., Townsend, E., Ness, J., Waters, K., \& Cooper, J. (2015). Hospital management of suicidal behaviour and subsequent mortality: a prospective cohort study. The lancet. Psychiatry, 2(9), 809-816.

https://doi.org/10.1016/S2215-0366(15)00169-8

[13] Hayashi, N., Igarashi, M., Imai, A. et al.. Posthospitalization course and predictive signs of suicidal behavior of suicidal patients admitted to a psychiatric hospital: a 2-year prospective follow-up study. BMC Psychiatry 12, 186 (2012).

https://doi.org/10.1186/1471-244X-12-186

[14] Hofstra, E., van Nieuwenhuizen, C., Bakker, M., Özgül, D., Elfeddali, I., de Jong, S. J., \& van der Feltz-Cornelis, C. M. (2020). Effectiveness of suicide prevention interventions: A systematic review and metaanalysis. General hospital psychiatry, 63, 127-140. https://doi.org/10.1016/j.genhosppsych.2019.04.011 